\title{
Fatal piglets diarrhea caused by a novel Chromobacterium haemolyticum
}

\author{
Leyi Zhang ${ }^{1}$, peng zhang ${ }^{1}$, Ge $\mathrm{Xu}^{1}$, Tairun Liang ${ }^{1}$, Jiawei Peng ${ }^{1}$, Shoutang $\mathrm{Wu}^{1}$, Ningjia \\ Yang $^{1}$, Lin Wang ${ }^{1}$, Yanling Liu ${ }^{1}$, Xinming Zhang ${ }^{1}$, Zheng $\mathrm{Xu}^{1}$, and Changxu Song ${ }^{1}$ \\ ${ }^{1}$ South China Agricultural University College of Animal Science
}

February 14, 2022

\begin{abstract}
Chromobacterium haemolyticum is a fatal gram-negative bacterium, which had been found that has the ability to infect human. In this study, we detected a nonpigmented, $\beta$-hemolytic gram-negative bacillus from fecal samples of piglets with severe diarrhea and death. Analysis of the 16S rRNA gene sequence indicated it was most closely related to Chromobacterium haemolyticum, however only with $79 \%$ identities, indicating that this bacterium may be a new type of Chromobacterium haemolyticum. Diarrhea was reproduced in experimental pigs with one of the isolates strains, designated GDHYZ30 strain. Intramuscular injection of the GDHYZ30 strain can cause death of the piglets, and irrigated challenge induced piglets diarrhea. To further elucidate the mechanism of pathogensis of this bacterium, whole genome sequencing was used to analyze and annotate the GDHYZ30 strain. The full-length genome of the strain was $4785117 \mathrm{bp}$, the $\mathrm{G}+\mathrm{C}$ content was $62.67 \%$, and it encoded 4398 genes, in which 57 drug resistance genes, and 390 virulence factors were included. It is worth noting that this novel Chromobacterium haemolyticum has been isolated from several other pig farm water samples and pig feces samples with unexplained diarrhea. To our knowledge this is the first report that a novel Chromobacterium haemolyticum as a new pathogen causing diarrhea and death in piglets and transmiting through water sources. And the genetic characteristics of the bacteria are analyzed by whole gene sequencing, which provides a reference for the prevention of human and animal infections and food safety.
\end{abstract}

\section{Fatal piglets diarrhea caused by a novel Chromobacterium haemolyticum}

Author names:

Leyi zhang ${ }^{1,2 \#}$, Pengfei Zhang ${ }^{1,2 \#}$, Ge Xu ${ }^{1,2}$, Tairun Liang ${ }^{1,2}$, Jiawei Peng ${ }^{1,2}$, Shoutang Wu ${ }^{1,2}$, Ningjia Yang $^{1,2}$, Lin Wang ${ }^{1,2}$, Yanling Liu ${ }^{1,2}$, Xinming Zhang ${ }^{1,2}$, Zheng $\mathrm{Xu}^{1,2 *}$, and Changxu Song ${ }^{1,2 *}$

1 College of Animal Science \& National Engineering Center for Swine Breeding Industry, South China Agriculture University, Guangzhou 510642, China;

2 Guangdong Laboratory for Lingnan Modern Agriculture, College of Animal Science, South China Agricultural University, Guangzhou 510642, China

*Corresponding author: Zheng $\mathrm{Xu}$ and Changxu Song

College of Animal Science \& National Engineering Center for Swine Breeding Industry,

South China Agriculture University, Guangzhou 510642, China

E-mail: cxsong2004@163.com \& cxsong@scau.edu.cn; Tel: +86-13829723528

\# These authors contributed equally to this work.

Abstract 
Chromobacterium haemolyticum is a fatal gram-negative bacterium, which had been found that has the ability to infect human. In this study, we detected a nonpigmented, $\beta$-hemolytic gram-negative bacillus from fecal samples of piglets with severe diarrhea and death. Analysis of the $16 \mathrm{~S}$ rRNA gene sequence indicated it was most closely related to Chromobacterium haemolyticum, however only with $79 \%$ identities, indicating that this bacterium may be a new type ofChromobacterium haemolyticum. Diarrhea was reproduced in experimental pigs with one of the isolates strains, designated GDHYZ30 strain. Intramuscular injection of the GDHYZ30 strain can cause death of the piglets, and irrigated challenge induced piglets diarrhea. To further elucidate the mechanism of pathogensis of this bacterium, whole genome sequencing was used to analyze and annotate the GDHYZ30 strain. The full-length genome of the strain was $4785117 \mathrm{bp}$, the $\mathrm{G}+\mathrm{C}$ content was $62.67 \%$, and it encoded 4398 genes, in which 57 drug resistance genes, and 390 virulence factors were included. It is worth noting that this novel Chromobacterium haemolyticum has been isolated from several other pig farm water samples and pig feces samples with unexplained diarrhea. To our knowledge this is the first report that a novelChromobacterium haemolyticum as a new pathogen causing diarrhea and death in piglets and transmiting through water sources. And the genetic characteristics of the bacteria are analyzed by whole gene sequencing, which provides a reference for the prevention of human and animal infections and food safety.

\section{Keywords: novel Chromobacterium haemolyticum; diarrhea; fatal; piglets}

\section{Introduction}

The genus Chromobacterium, consisting of seven recognized species, is a Gram-negative, rod-shaped bacterium, in which the most famous specie is Chromobacterium violaceum with red pigment (Papapanagiotou \& Aligizakis, 1959). Chromobacterium is mainly distributed in tropical and subtropical regions, and it is frequently isolated in water sources and humid areas (Blackburn et al., 2019). Chromobacterium haemolyticum was considered as a separate species, lacking violet pigmentation but demonstrating strong hemolysis on sheep blood agar. (Han, Han, \& Segal, 2008). C .haemolyticum has been isolated in a variety of water environments, such as rice roots, lakes, rivers, and sewage effluent (Kanamori et al., 2020; Priya, Sulaiman, How, Yin, \& Chan, 2018; Zhou et al., 2016). There is evidence that $C$. haemolyticum is mainly transmitted through water sources, and some reports suggest that $C$. haemolyticum can cause pneumonia and sepsis in humans, and the patients in these cases have a history of wound exposure or contact with water sources, indicating that Chromobacterium haemolyticum maybe an opportunistic pathogen (Miki \& Okada, 2014; Takenaka et al., 2015). There have also reports that diarrhea case was related to $C$. haemolyticum, but $C$.haemolyticum causing diarrhea or even death in other animals has not been reported (Tanpowpong, Charoenmuang, \& Apiwattanakul, 2014).

Piglets diarrhea is a very severe disease that is very harmful to the breeding industry. The intestinal immunity of piglets is not fully developed, and many pathogens are susceptible to piglets at this stage, causing acute diarrhea, vomiting, dehydration, and death (Osek \& Truszczynski, 1992; Wang, Vlasova, Kenney, \& Saif, 2019). Diarrhea in piglets can be devastating to pig farms if the main pathogen cannot be identified and treated accordingly. Some farms use mountain springs or river water as water sources, and incomplete disinfection of water sources often leads to infection of some bacterial diseases. C .haemolyticum is usually detected in water sources. Although it is pathogenic, it has received less attention. It is necessary to conduct in-depth research on the biological characteristics and genetic characteristics of the bacteria.

This study found that unexplained diarrhea occurred in many piglet farms in south China. After excluded some regular pathogens, a novel type ofChromobacterium haemolyticum was found in the water source and fecal samples of piglets with diarrhea. Challenge piglets with isolate GDHYZ30 can cause the same diarrhea symptoms similar to that of the field cases. And the diarrhea of piglets had been controlled after changing the drinking water source, indicating that the GDHYZ30 strain is the agents that cause diarrhea and death in piglets.

\section{Materials and methods}

In this study, no regular pathogens causing piglet diarrhea were detected in feces and drinking water samples 
of diarrheal piglets collected in farm areas where diarrhea occurred, but a $\beta$-hemolytic bacterium was found, which was also isolated in the drinking water. Subsequently, 16SrRNA primers were used to amplify and sequence, and the results showed that the bacteria were a $C$. haemolyticum like bacterium by phonotype. The bacteria isolated were used for the challenge trial. Nine 30-day-old healthy piglets were randomly divided into three groups. $2 \mathrm{ml}$ of the $3.5 \times 10^{\wedge} 9$ colony forming unit (CFU) of the bacterium were administered orally or intramuscularly injected respectively, and $2 \mathrm{ml}$ of PBS was administered orally to the control group. The isolate GDHYZ30 could cause the same diarrhea symptoms similar to that of the clinical cases when the piglets were challenged. To understand the genetic characteristics of the bacteria, whole gene sequencing was Completed by Sangon Biotech Company. This whole-genome sequencing project has been deposited at GenBank under the accession number: PRJNA802706.

\section{Results}

\section{Clinical and biological properties}

Our investigation found severe piglet diarrhea in one farm. The morbidity rate of piglets was $30.65 \%$ $(149 / 486)$, and the mortality rate was $35.57 \%$ (53/149). To figure out the agents of diarrhea in piglets, we collected fecal samples from piglets with diarrhea and tested a variety of diarrhea-causing viruses including Porcine epidemic diarrhea virus; Porcine deltacoronavirus; Transmissible Gastroenteritis Virus; swine acute diarrhoea syndrome coronavirus; porcine rotavirus; Porcine circovirus type 3 and Porcine Circovirus-like Viruses by PCR methods, all the above pathogens could not be detected. Excitingly, a $\beta$-hemolysis bacterium was isolated (Fig.1A), which were Gram-stained negative, rod-shaped bacterium (Fig.1B). The strain was purified by colonization, and named as GDHYZ30 strain. Subsequently, Koch postulates experiments were conducted to verify the pathogenicity of the GDHYZ30 isolate. Nine 30-day-old healthy piglets were randomly divided into three groups. $2 \mathrm{ml}$ of $3.5 \times 10^{\wedge} 9 \mathrm{CFU}$ bacteria were administered orally or intramuscularly injected respectively, and $2 \mathrm{ml}$ of PBS was administered orally to the control group. At the second day, we found that all three piglets in the gavage group developed diarrhea (Fig.1C). One piglet in the intramuscular injection group had diarrhea and two piglets died. Piglets in the control group were asymptomatic. Piglets with diarrhea had thin intestinal walls and flatulence (Fig.1D). The GDHYZ30 strain could be isolated from the blood and heart of the dead piglets, but not from the piglets administered orally group. The above results indicated that the GDHYZ30 strain was the pathogen causing piglet diarrhea and acute death.

\section{Genetic evolution and prevalence}

In order to study the genetic characteristics of the bacterium, we carried out the whole gene sequencing analysis of the bacillus. The 16SrRNA phylogenetic tree analysis showed that the bacterium belongs toChromobacterium, which is in the same branch asChromobacterium haemolyticum, with a homology of $79 \%$ (Fig. 2A). The climatic characteristics of Guangdong Province are more suitable for the reproduction of Chromobacterium, so we expanded the detection range. The Chromobacterium haemolyticum was also detected in water sources and fecal samples in other areas with unexplained diarrhea. The bacteria were detected in the three regions of HEYUAN, MAOMING, and SHANWEI, among which the incidence was more severe in HEYUAN region (Fig.2B). These results suggest that the GDHYZ30 strain should be noteworthy to focus on in south China, since this novelChromobacterium haemolyticum could be an important agent causing diarrhea and death in piglets, and a potential infection risk factor for human beings by food chain as well.

\section{Genome annotation}

Whole gene sequencing showed that the genome size of strain GDHYZ30 is 4,785,117 bp, with 4,398 protein coding genes and the genome's GC content is $62.67 \%$ and the longest protein coding gene is 11,694 bp. The average length of genes encoding proteins is $957 \mathrm{bp}$, the proportion of genes encoding proteins was 88.04\% (Fig.3A\&Table 1). After comparing the reference sequences using BLAST x against NR (NCBI nonredundant protein sequences), Swiss-Prot, COG (Clusters of Orthologous Groups of proteins, and KEGG (Kyoto Encyclopedia of Genes and Genomes) databases, we identified 4220 unigenes providing a significant 
result in NR, and 3179, 3346, and 2145 unigenes were annotated according to Swiss-Prot, COG, and KEGG databases, respectively (Fig.3B). By aligning with the NR library, it is possible to view the approximation of the transcript sequence of the species and the similar species, as well as the functional information of the homologous sequence. GDHYZ30 strain had the most sequences aligned with the Chromobacterium haemolyticumstrain with 3059 sequences (Fig.3C). Functional prediction and classification of unigenes was performed by comparing sequence data against the COG database. A total of 1935 unigenes were annotated and grouped into14 categories according to COG function classifications. Among them, the top 3 clusters for general function prediction were "amino acid transport and metabolism" (330 genes), "transcription" (262 genes); , and "Energy production and conversion" (191 genes) (Fig.3D).

\section{Virulence genes and drug resistance}

The analysis of drug resistance genes found that the GDHYZ30 strain had 57 drug resistance genes, which were resistant to $\beta$-lactams, carbapenem hydrolyzing $\beta$-lactamases, etc. (Table 2). GDHYZ30 was compared with SETA and SETB databases respectively, the GDHYZ30 strain was matched with 390 virulence factors in the seta database and 432 virulence factors in the seta database (Table 3). The analysis of drug resistance genes showed that the bacteria expressed 57 kinds of drug resistance genes and had drug resistance to $\beta$ lactams and carbapenem hydrolyzing $\beta$-lactamases etc. These results provide a reference for the study of the treatment and pathogenesis of the disease.

\section{Discussion}

Our investigation found outbreaks of diarrhea and death in piglets drinking mountain spring water. We isolated a novel hemolytic Gram-negative bacterium in the fecal samples and drinking water of piglets with diarrhea and named GDHYZ30. 16SrRNA analysis demonstrate that the bacteria is close to Chromobacterium haemolyticum. Then we designed the Koch postulates experiments to confirm that the GDHYZ30 strain is the causative agent of piglet diarrhea and death. Then the whole gene sequencing analysis of the GDHYZ30 strain was carried out. The phylogenetic tree based on 16SrRNA showed that the GDHYZ30 strain belongs to the same branch as Chromobacterium haemolyticum, but the homology is only $79 \%$, indicating that this strain may belong to a new group, mainly causing diseases characterized by acute diarrhea and death. It has been reported that Chromobacterium haemolyticum has anti-bacterial properties, and we speculate that infection withChromobacterium haemolyticum, except of the direct intestinal damage, may cause changes in the intestinal flora, reducing beneficial bacteria and leading diarrhea consequently as well. (Zwe et al., 2021). At the same time, we found that infection with this novelChromobacterium haemolyticum can cause death, and intramuscular injection of the GDHYZ30 strain even can cause acute death of piglets. Meanwhile, the GDHYZ30 strain could be isolated from the blood samples and hearts of dead pigs, while the gavage group only caused diarrhea. These results suggest that bacteria can cause bacteremia leading to acute death, mainly through wound infection, and the results are similar to the way humans are infected with Chromobacterium haemolyticum .(Harmon, Mortensen, Robinette, \& Powell, 2016; Miki \& Okada, 2014). The bacteria have 390 virulence genes and 57 drug resistance genes, whether these genes are pathogenic still needs further research. Clinical trials and drug resistance analysis show that this novel Chromobacterium haemolyticum is a potential threat to humans and animals. The suitable conditions for the growth ofChromobacterium haemolyticum are near moist water sources, and the geographical location and climate of Guangdong Province are very suitable for the reproduction of Chromobacterium haemolyticum(LimaBittencourt, Costa, Barbosa, Chartone-Souza, \& Nascimento, 2011; Priya et al., 2018). We also detected Chromobacterium haemolyticum in the drinking water of other pig farms with unexplained diarrhea, and after changing the water source, the piglet diarrhea was controlled. Therefore, checking the sanitation of water sources can prevent the occurrence of the disease.

Conflicts of interest

All authors declare that they have no conflicts of interest.

Acknowledgements 
Funding: This study was supported by the National Key Research and Development Program of China (2018YFD0501102), the Key-rea Research and Development Program of Guangdong Province (2019B020211003).

\section{Figure legends}

Fig.1 Bacterial isolation and piglets challenge. (A) $\beta$-hemolysis on sheep blood agar; (B) Gram Staining; (C) Diarrhea in challenge piglets; (D) Intestinal lesions in piglets with diarrhea.

Fig.2 Phylogenetic relationship and epidemiological. (A)Neighbour-joining phylogenetic tree based on a comparison of the $16 \mathrm{~S}$ rRNA gene sequences of strain GDHYZ30 strain and its closest relatives Chromobacterium strains; (B) Regions of Chromobacterium haemolyticum infection in Guangdong province. The region affected withChromobacterium haemolyticum was indicated in pink.

Fig.3 Whole gene annotation. (A) Distribution of gene lengths encoding proteins; (B) Venn diagram of annotated gene function among KEGG, KOG, NR, and Swiss-prot; (C) Comparison of homologous genes in NR databases; D COG functional classification of the GDHYZ30.

Table.1 Genomic sequencing information.

Table.2 Drug resistance gene statistic.

Table.3 Virulence factor statistics.

\section{References}

Blackburn, M. B., Farrar, R. R., Jr., Sparks, M. E., Kuhar, D., Mowery, J. D., Mitchell, A., \& GundersenRindal, D. E. (2019). Chromobacterium phragmitis sp. nov., isolated from estuarine marshes. Int J Syst Evol Microbiol, 69 (9), 2681-2686. doi:10.1099/ijsem.0.003508

Han, X. Y., Han, F. S., \& Segal, J. (2008). Chromobacterium haemolyticum sp. nov., a strongly haemolytic species. Int J Syst Evol Microbiol, 58 (Pt 6), 1398-1403. doi:10.1099/ijs.0.64681-0

Harmon, N., Mortensen, J. E., Robinette, E., \& Powell, E. A. (2016). Pediatric bacteremia caused by Chromobacterium haemolyticum/Chromobacterium aquaticum. Diagn Microbiol Infect Dis, 86 (1), 108111. doi:10.1016/j.diagmicrobio.2016.05.021

Kanamori, H., Aoyagi, T., Kuroda, M., Sekizuka, T., Katsumi, M., Ishikawa, K., . . . Kaku, M. (2020). Chromobacterium haemolyticum Pneumonia Associated with Near-Drowning and River Water, Japan.Emerg Infect Dis, 26 (9), 2186-2189. doi:10.3201/eid2609.190670

Lima-Bittencourt, C. I., Costa, P. S., Barbosa, F. A., Chartone-Souza, E., \& Nascimento, A. M. (2011). Characterization of a Chromobacterium haemolyticum population from a natural tropical lake. Lett Appl Microbiol, 52 (6), 642-650. doi:10.1111/j.1472-765X.2011.03052.x

Miki, T., \& Okada, N. (2014). Draft Genome Sequence of Chromobacterium haemolyticum Causing Human Bacteremia Infection in Japan. Genome Announc, 2 (6). doi:10.1128/genomeA.01047-14

Osek, J., \& Truszczynski, M. (1992). Occurrence of fimbriae and enterotoxins in Escherichia coli strains isolated from piglets in Poland. Comparative immunology, microbiology and infectious diseases, 15 (4), 285-292. doi:10.1016/0147-9571(92)90008-f

Papapanagiotou, J., \& Aligizakis, C. (1959). Isolation of Chromobacterium prodigiosum from empyema. $J$ Clin Pathol, 12 (2), 170-171. doi:10.1136/jcp.12.2.170

Priya, K., Sulaiman, J., How, K. Y., Yin, W. F., \& Chan, K. G. (2018). Production of N-acyl homoserine lactones by Chromobacterium haemolyticum KM2 isolated from the river water in Malaysia. Arch Microbiol, 200 (7), 1135-1142. doi:10.1007/s00203-018-1526-y 
Takenaka, R., Nureki, S., Ueno, T., Shigemitsu, O., Miyazaki, E., Kadota, J., . . . Okada, N. (2015). Chromobacterium haemolyticum Pneumonia Possibly Due to the Aspiration of Runoff Water. Jpn J Infect Dis, 68 (6), 526-529. doi:10.7883/yoken.JJID.2014.285

Tanpowpong, P., Charoenmuang, R., \& Apiwattanakul, N. (2014). First pediatric case of Chromobacterium haemolyticum causing proctocolitis.Pediatr Int, 56 (4), 615-617. doi:10.1111/ped.12301

Wang, Q. H., Vlasova, A. N., Kenney, S. P., \& Saif, L. J. (2019). Emerging and re-emerging coronaviruses in pigs. Current Opinion in Virology, 34, 39-49. doi:10.1016/j.coviro.2018.12.001

Zhou, S., Guo, X., Wang, H., Kong, D., Wang, Y., Zhu, J., . . . Ruan, Z. (2016). Chromobacterium rhizoryzae sp. nov., isolated from rice roots.Int J Syst Evol Microbiol, 66 (10), 3890-3896. doi:10.1099/ijsem.0.001284

Zwe, Y. H., Yadav, M., Zhen Ten, M. M., Srinivasan, M., Jobichen, C., Sivaraman, J., \& Li, D. (2021). Bacterial Antagonism of Chromobacterium haemolyticum and Characterization of its Putative Type VI Secretion System. Res Microbiol, 103918. doi:10.1016/j.resmic.2021.103918

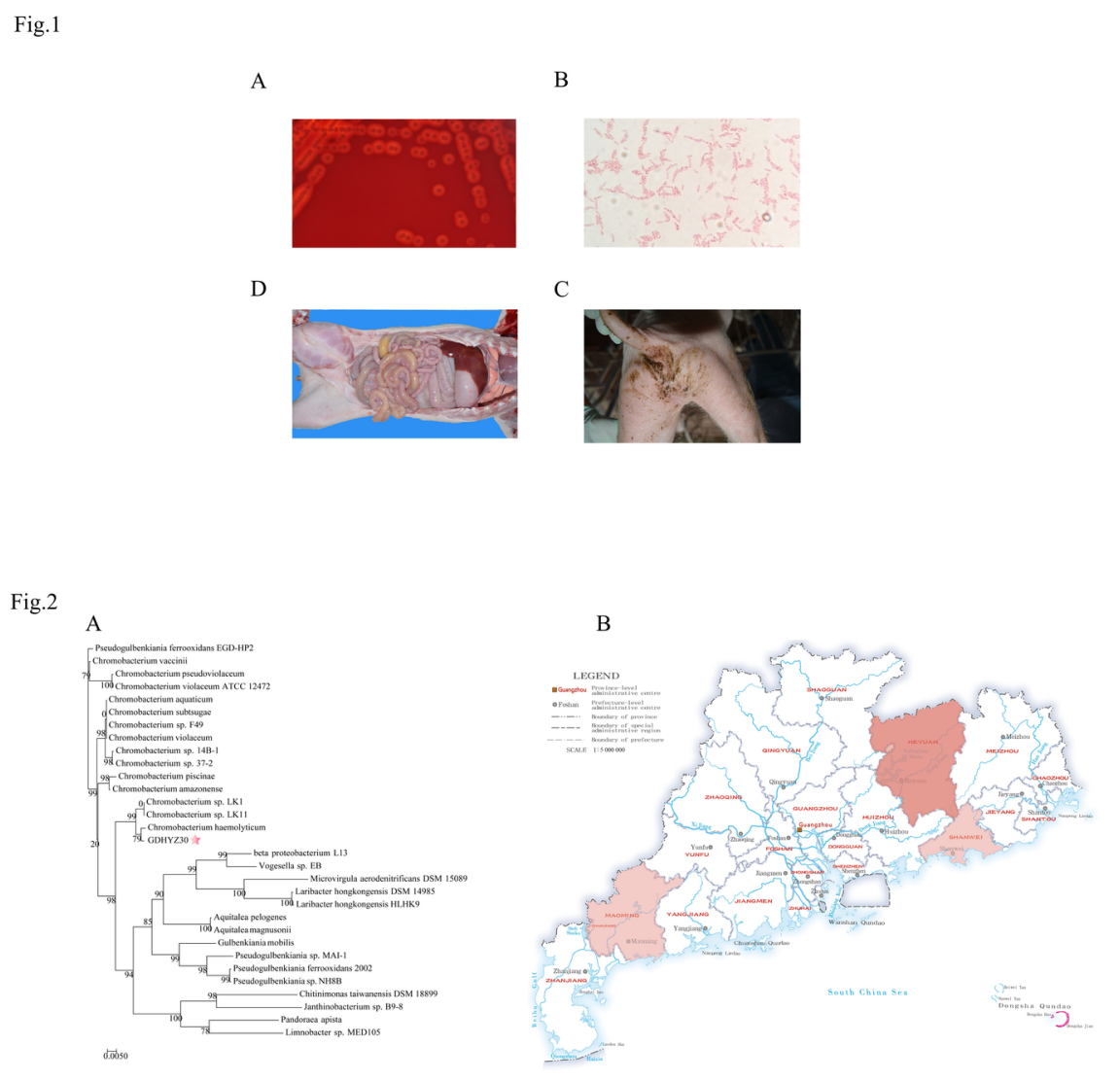




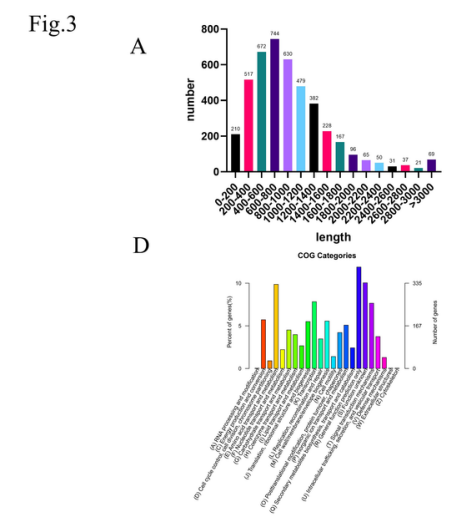

B

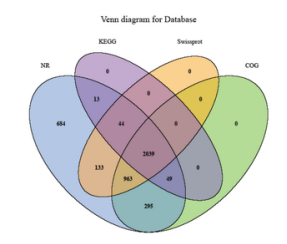

C

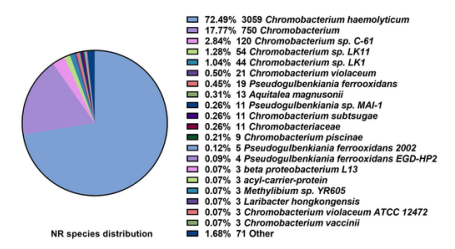

\section{Hosted file}

Table.1.xls available at https://authorea.com/users/370590/articles/556392-fatal-pigletsdiarrhea-caused-by-a-novel-chromobacterium-haemolyticum

\section{Hosted file}

Table.2.xlsx available at https://authorea.com/users/370590/articles/556392-fatal-pigletsdiarrhea-caused-by-a-novel-chromobacterium-haemolyticum

\section{Hosted file}

Table.3.xlsx available at https://authorea.com/users/370590/articles/556392-fatal-pigletsdiarrhea-caused-by-a-novel-chromobacterium-haemolyticum 